\title{
Procedimiento metodológico de auditoría interna para la Cooperativa de Ahorro y Crédito Santiago Ltda., del cantón Loja
}

Internal Audit Methodology in the Savings and Loan Association "Santiago Ltda." of the Canton Loja

Wilman Patricio Pacheco Poma. ${ }^{1}$, Cecilia Ivonne Narváez Zurita. ${ }^{2}$, \& Juan Carlos Erazo Álvarez. ${ }^{3}$

\section{DOI: https://doi.org/10.33262/visionariodigital.v3i2.1.557}

\begin{abstract}
.
Nowadays, Internal audit is not just limited to analyze financial accounting elements but also looks for quality assurance and operational improvement of an organization. In this sense, this research work proposes to design an Internal Audit methodology for the Savings and Loan Association "Santiago Ltda," located in the canton of Loja, to meet its aims through the assessment and improvement of operational effectiveness process, control, risk management, and corporate governance.

The International Standards on Auditing (ISAS), the provisions of the Superintendence of Popular and Solidarity Economy and the Financial Analysis Unit (UAF) are framing this proposal. Since the internationalization and the economic complexity of financial institutions, particularly savings and loan cooperatives, has demanded a new refocus and specialization in terms of financial intermediation. This research is descriptive and explanatory supported by a theoretical and doctrinal source. The information was gathering through surveys to the executive team, heads of the analysis unit, which was evaluated and interpreted for the subsequent

${ }^{1}$ Universidad Católica de Cuenca, Posgradista Maestría en Contabilidad y Auditoría, Cuenca, Ecuador, wppachecop583@psg.ucacue.edu.ec

${ }^{2}$ Universidad Católica de Cuenca, Subdirección de Posgrado, Cuenca, Ecuador,

${ }^{3}$ Universidad Católica de Cuenca, Subdirección de Posgrado, Cuenca, Ecuador,
\end{abstract} inarvaez@ucacue.edu.ec jcerazo@ucacue.edu.ec 
presentation of the results. Finally, the paper concludes that through the effective implementation of this methodology, the timely detection of mistakes will support to apply corrective and preventive actions to enhance the current association situation.

Keywords: Internal Audit, Accounting Control, Administrative Control, Popular and Solidarity Economy, Risk Management.

\section{Resumen.}

La auditoría interna en la actualidad no se limita al análisis de elementos contables financieros sino que busca el aseguramiento y mejora de las operaciones de una organización. En este sentido, en la presente investigación se propone el diseño de un procedimiento metodológico de auditoría interna para la Cooperativa de Ahorro y Crédito "Santiago Ltda", del cantón Loja, a efectos de aportar al cumplimiento de sus objetivos mediante la evaluación y mejora de la eficacia de sus procesos operativos, de control, de gestión de riesgos y gobierno corporativo.

Los procedimientos que rigen sobre esta propuesta están enmarcados en las Normas Internacionales de Auditoría (NIA), en las disposiciones de la Superintendencia de Economía Popular y Solidaria (SEPS) y de la Unidad de Análisis Financiero (UAF), ya que la internacionalización y la complejidad económica de las entidades financieras y de manera especial de las cooperativas de ahorro y crédito, ha exigido su redimensionamiento y especialización en lo referente a la intermediación financiera. En tal razón, la investigación fue descriptiva- explicativa ya que se realizó el análisis de la información teórica y doctrinaria relacionada con el tema planteado, para el levantamiento de la información se aplicaron encuestas a nivel de directivos y funcionarios de la unidad de análisis, la misma que fue procesada, analizada e interpretada para la posterior presentación de resultados. Finalmente se concluyó que con la efectiva aplicación del procedimiento metodológico se podrá detectar oportunamente los errores e implementar medidas correctivas y/o preventivas para mejorar la situación actual de la Cooperativa.

Palabras claves: auditoría interna, cooperativas de ahorro y crédito, control contable, control administrativo, economía popular y solidaria

\section{Introducción.}

En la Constitución de la República del Ecuador (2008) la economía popular y solidaria se reconoce y distingue como un pilar fundamental del sistema económico del País, teniendo como principios fundamentales la solidaridad, reciprocidad y cooperación, está conformado por el sector financiero y no financiero que lo integran las cooperativas, asociaciones, organizaciones comunitarias y mutualistas. En lo que respecta al sector financiero, las 
cooperativas de ahorro y crédito, de acuerdo a la Norma para la segmentación de las entidades del sector financiero popular y solidario se distribuyen en 5 segmentos de acuerdo al tipo y saldo de sus activos, y se encuentran controlados por la Superintendencia de Economía Popular y Solidaria (SEPS).

La instauración del sistema económico popular y financiero en la sierra ecuatoriana ha permitido una mayor vinculación con los sectores sociales excluidos, dentro de la Provincia de Loja el sector popular y solidario se encuentra en vías de desarrollo y actualmente está conformado por 45 cooperativas de ahorro y crédito distribuidas en los diferentes cantones y parroquias, las cuales ofrecen una variedad de productos y servicios con el firme propósito de satisfacer las necesidades pero sobre todo ayudar económicamente a sus socios y clientes.

En el medio económico actual en donde se desenvuelven las cooperativas de ahorro y crédito, es importante que los directivos implementen la mayor cantidad de herramientas de control de sus recursos, logrando con ello, su optimización y que el logro de sus metas sea vea reflejado en el cumplimiento estricto de las disposiciones legales emitidas para el efecto. Al ser las cooperativas de ahorro y crédito parte del sector financiero nacional, y que día a día refleja un flujo de efectivo importante, la administración debe estar en todo momento en un control total de sus disponibilidades y demás rubros presentes, de ahí que los procedimientos de auditoría interna se convierten en una estrategia que les permite prevenir situaciones adversas que amenacen su desarrollo y sirve de prevención para futuras intervenciones de agentes externos en la evaluación de la información económica; su aplicación asegura que todos los departamentos cuenten con los requisitos e insumos necesarios para desempeñarse de manera eficiente, lo cual contribuye al bienestar de sus socios y ciudadanía en general.

La auditoría interna es una herramienta básica e indispensable para que todas las instituciones dedicadas a la intermediación financiera puedan tener un control eficiente sobre sus actividades cotidianas que se ven reflejadas en sus estados financieros con una información financiera sólida y confiable, lo que coadyuva al logro de sus metas perfiladas, con un apego estricto de las disposiciones legales, convirtiéndose entonces en una de las mayores estrategias implementadas por las organizaciones para el aseguramiento de su información financieras y para dar cumplimiento a las normativas legales vigentes.

Ballesteros (2009) menciona que la importancia de la auditoría en las empresas radica precisamente en que se convierte en un mecanismo de control para contribuir a alcanzar metas, satisfacción del cliente y sobre todo fidelidad, y a consecuencia de ello, lograr los objetivos de rentabilidad esperados por todos los socios de la organización. A la auditoría no se la debe tomar como sancionadora, sino más bien como preventiva y cuyos resultados 
permiten que los propietarios de las empresas examinadas, puedan tomar los correctivos necesarios para mejorar sus aspiraciones empresariales.

\section{Antecedentes de la Auditoría}

Viloria (2004) manifiesta que los orígenes de la auditoría son remotos, ya que el hombre no solo sintió la necesidad de registrar sus operaciones sino que también quería tener un dominio sobre ellas, y es así que con el paso del tiempo la auditoría pasó de "oír" a la emisión de una opinión sobre los estados financieros. En este sentido, la auditoría ha servido a las empresas desde tiempos remotos, y se ha ido afianzando y mejorando su accionar en concordancia con la evolución de las otras ciencias, razón de ello, es que ahora se conocen una gran variedad de tipos de auditorías, que ha surgido a raíz de la necesidad de conocer ciertos asuntos específicos que no se podían realizar con un solo procedimiento en particular, fortaleciendo su campo de acción y su servicio a los sectores públicos y privados.

Con el pasar de los años la auditoría ha evolucionado exigiendo la presentación de documentos que reflejen las operaciones y evidencias de las técnicas de teneduría de libros, sustentándose en la quiebra inesperada de varias organizaciones importantes de la época (Villardefrancos, Álvarez y Rivera, 2006). De ahí que la tendencia de la auditoría está encaminada a fortalecer la evaluación sobre la razonabilidad de los estados financieros a fin de tener certeza absoluta del eficiente manejo de los recursos que se asignan a una producción específica. Dentro del campo de la auditoría, se pueden encontrar varias clasificaciones, dependiendo de la profundidad y alcance que tendrá la misma; cabe mencionar que, la financiera ocupa un lugar vital en razón que es la precursora del campo evaluativo. En la tabla 1, se detallan los diferentes tipos de auditoría que cubren o satisfacen las necesidades institucionales.

Tabla 1. Tipos de auditoría

\begin{tabular}{|c|c|}
\hline Auditoría & Objetivo Principal \\
\hline Fiscal & $\begin{array}{l}\text { Evaluar el cumplimiento de la normativa tributaria del Servicio de Rentas } \\
\text { Internas (SRI). }\end{array}$ \\
\hline Operacional & $\begin{array}{l}\text { Supervisar el desarrollo de operaciones y procesos institucionales, basados } \\
\text { en la eficiencia. }\end{array}$ \\
\hline Administrativa & $\begin{array}{l}\text { Evaluar la administración general aplicada a las diversas operaciones } \\
\text { realizadas por la empresa. }\end{array}$ \\
\hline Informática & Revisar y evaluar actividades funcionales de hardware y software. \\
\hline
\end{tabular}


Integral Evaluar los principios empresariales: eficiencia, eficacia, gestión y resultados.

Ambiental

Supervisar y medir los procesos e impactos ocasionados al medio ambiente en la práctica.

Calidad

Evaluar la calidad del proceso y del producto final, de acuerdo a la normativa vigente.

Social Evaluar el fiel compromiso empresarial con el entorno social y su bienestar general

Gubernamental Examinar diferentes operaciones en entidades y dependencias del Estado.

Fuente: Sotomayor, 2008.

En tal contexto, el acelerado desarrollo de la auditoría en analogía con el avance tecnológico ha conllevado al perfeccionamiento de las técnicas de control, definición del objeto de auditoria, sus métodos e instrumentos, independientemente de su clasificación se subdivide según la independencia de la persona que realiza el proceso de evaluación pudiendo ser interna cuando se realiza por personas dependientes de la organización y realiza estos procesos para agregar valor y mejorar las operaciones, en tanto la auditoría externa es realizada por entes de control y su función es determinar el cumplimiento estricto de las disipaciones legales.

La auditoría interna desde su concepción se enmarcaba en la "revisión de la eficiencia de los empleados, procedimientos administrativos, actualización de políticas" (Morales, 2012, p.10). La auditoría interna se constituye básicamente en un proceso interno realizado por un profesional con el único fin de coadyuvar al cumplimiento mismo de los objetivos primordiales de la organización, constituyéndose en una actividad objetiva para asegurar que las operaciones realizadas tengan un apego estricto al cumplimiento cabal de todas las normas, reglamentos y demás que estipulan la leyes vigentes, lo que se convertirá en un valor agregado para la empresa (Santillana, 2003).

Vega y Madelayne (2006) expresan que la auditoría interna es un proceso lógico y ordenado que a grandes rasgos pretende estudiar, descubrir, identificar y evaluar una situación en particular, su práctica se centra en los sistemas de control interno implementados por la empresa y su diagnóstico global. Según las tendencias actuales la auditoría interna se debe ocupar de identificar y gestionar los riesgos que por su naturaleza tiene la empresa y sobre todo buscar alternativas de solución accesibles para la organización. Entre las principales cualidades de la auditoría interna se encuentran: 
Naturaleza: radica en el análisis de su concepto, propósito y alcance que de manera precisa surgen de la necesidad de reforzar la organización, con la finalidad de disminuir el riesgo y proteger sus activos, (Tapia, Rueda y Silva, 2017). El Propósito es entregar a la alta gerencia de la organización resultados del análisis de sus operaciones internas con interacción a factores externos basados en leyes vigentes, y su alcance permite evaluar a todas las áreas de la empresa así como a todas sus operaciones, respecto de la veracidad y confiabilidad de la información representada en los estados financieros, todo esto en torno a las leyes y regulaciones vigentes (Santillana, 2013, p.10).

Por otra parte, dentro de los objetivos de la auditoría interna están los siguientes:

- Evaluar las normas y reglamentos que son aplicados apropiadamente por la organización para el cumplimiento de la ley.

- Evaluar el sistema de control interno aplicado, con el objeto de obtener un mejor rendimiento.

- Ayudar a la administración de la organización con información veraz del cumplimiento de funciones y compromisos contraído con sus socios, terceros y con la sociedad en general.

El campo de aplicación de la auditoría interna es extensa, por ello es necesario contar con normas que controlen y regulen la práctica de aplicación, y deben estar relacionadas directamente con las cualidades del profesional de auditoria puesto que de su experiencia dependerá la aplicación correcta y el resultado se verá plasmado en un trabajo de calidad.

Las normas de auditoría generalmente aceptadas o conocidas como NAGAS, según Tapia, Guevara, Castillo, Rojas y Salomón (2016) son el resultado del proceso de adopción de las Normas Internacionales de Auditoría (NIA) y se convierten en principios fundamentales en los que debe enmarcarse el desempeño de los auditores profesionales durante toda la ejecución de un proceso de auditoría ya sea interno o externo; como resultado y en base a la aplicación de las NAGAS se garantiza la calidad e integridad del trabajo profesional del auditor. Por su parte, Cardozo (2016) menciona que las NAGAS "se relacionan con las cualidades profesionales del contador público y con el empleo de su buen juicio en la ejecución de su examen y en su informe"(p.17), para ello se concidera relevante evaluar adecuadamente la estructura de cntril internos de las empresas, para si establecer el grado de confianza que merece, y consecuentemente establecer la narturalesza, alcance y la oportunidad de los procedimeitno de auditoria a implementar.

El control interno se puede definir como un vínculo entre normas, procedimientos y técnicas a las que debe regirse o tener como guía base dentro de las operaciones que desempeña la empresa, con la finalidad de corregir o advertir las amenazas que afectan el fiel cumplimiento 
de los objetivos trazados por la organización, adicionalmente mediante la implementación del control interno se puede garantizar la confiabilidad de la información obtenido como resultado de sus operaciones y por el giro mismo de la empresa. Es así que, Mantilla (2008) establece que "el control interno es diseñado e implementado por la administración para tratar los riesgos de negocios y de fraude identificados que amenazan el logro de los objetivos establecidos, tales como la confiabilidad de la información financiera"(p.12).

El control interno en su principal función ayuda al cumplimiento eficaz de los objetivos empresariales, para lo cual es necesario evaluar desde el punto de vista costo/beneficio (Catácora, 1996). En su publicación Coopers y Lybrand (1997) establecen que el control interno está conformado por 5 componentes interrelacionados:

1. Ambiente para el control

2. Apreciación de riesgos

3. Movimientos y actividades de control

4. Alineación y declaración

5. Verificación de resultados

\section{Auditoría de estados financieros}

Los estados financieros son la representación escrita del resultado final de las operaciones desarrolladas por una organización dentro de un ejercicio económico, por el cual se pone en evidencia mediante términos financieros y monetarios la situación real de su posicionamiento y operatividad financiera, con lo que ayudará a la alta gerencia a la toma de decisiones oportunas y reales (Bravo, 2011). En tal contexto, Osorio (2000) y Mendebil (2013) manifiestan que la auditoría de los estados financieros se resume a una evaluación general de la información generada durante un hecho económico, que está basado en normas, técnicas y procedimientos efectuados por un profesional afín, quien como resultado de su evaluación emitirá un documento llamado dictamen.

Al respecto, Guzmán y Verdezoto (2015) definen a la auditoría financiera como una revisión, inspección, examen objetivo, profesional y sistemático, y si a esto se le suma el término financiero, se enfatiza en un estudio a los estados financieros de una empresa en particular, realizado con posterioridad al cierre del ejercicio contable y cuyo fin es emitir un juicio profesional para determinar la razonabilidad de los saldos o afirmaciones presentadas por la administración de la empresa.

En el caso de las cooprativas de ahorro y credito constituidas en el Ecuador, los estados financieros deben estructurarse bajo el catálogo único de cuentas (CUC) emitido por la Superintendencia de Economía Popular y Solidaria (SEPS), dentro del cual se plasma su 
desarrollo en base a 8 dígitos de acuerdo a la información que se pretende identificar: grupo, sub grupo, cuentas y sub cuentas, entre las principales cuentas que se identifican en CUC se encuentran las siguientes:

Fondos disponibles: son los recursos físicos con los que cuenta la organización, de alta liquidez para el desarrollo normal de sus operaciones, se encuentran disponibles y son de alta solvencia y dinamismo. Provienen de ingresos generados por servicios y depósitos, para su control se pueden ejecutar arqueos físicos, planillas, conciliaciones, evaluación de reportes diarios, etc.

Cartera de crédito constituida por el detalle de los valores que la organización ha entregado a sus socios y a terceros en calidad de préstamos o inversiones, más los intereses que la ley establece para este tipo de intermediación financiera, dentro de este grupo de cuentas también se considera los rubros de créditos vencidos. Para su clasificación se toma en consideración el nivel de riesgo que presenten de acuerdo a sus valores, modalidades, amortización y garantías, así como la evaluación del potencial acreedor en cuanto a su capacidad de pago, solvencia y situación crediticia

Cuentas por cobrar: representan los estipendios a cobrar por el resultado de la prestación de servicios que ofrece la organización, recuperación de anticipos y demás sumas por cobrar percibidas por cualquier otro concepto, como instrumentos de cobro se puede establecer las letras de cambios, títulos de crédito y pagares. Provisión de cuentas incobrables. Para salvaguardar las cuentas pendientes de cobro se destina un porcentaje de la misma, a fin de que si se convierten en incobrables al final de ejercicio económico puedan ser consideradas como gastos.

Propiedad planta y equipo: Se puede definir como propiedad planta y equipo a todos los activos tangibles de la entidad, que provienen como resultado de sus operaciones, son utilizados para el giro mismo del negocio, y son empleados a largo plazo (Zapata y Aymara, 2010). Su representación dentro de los estados financieros se guía directamente a reflejar la inversión que la organización ha efectuado para poder desarrollar su actividad, de igual forma el decremento sufrido por depreciaciones, pérdidas, daños, etc. con una incidencia notable dentro del patrimonio.

Otros activos: Dentro de este grupo se encuentran todos los bienes con que cuenta la organización y que no se encuentran integrados en los grupos anteriormente descritos, o no tienen cuenta individual asignada, debido a su naturaleza o su comportamiento financiero, se registran valores no tangibles o por percepción de otros pagos y es necesario para su valoración la aplicación de un método de valoración técnica. (Fierro, 2015, p.115) 
Obligaciones con el público: representa las obligaciones con los socios y terceros, debido al depósito de efectivo, cheques y demás que representan un valor monetario y que son susceptibles de cancelar efectivo (Fierro, 2016). Estas cuentas tienen una trascendental importancia puesto que permite el desarrollo normal del giro mismo de la organización o intermediación financiera, ya que son cuentas de mayor susceptibilidad se deben desarrollar planes, estrategias y políticas que puedan garantizar la licitud e integridad de estos ingresos

Cuentas por pagar: registra valores originados por conceptos de interés de las obligaciones con el público, pago de impuestos, contribuciones, pago a empleados y aportes al IESS, multas, pago de proveedores y terceros. Estas cuentas tienen carácter transitorio y no pueden sobrepasar más allá de los 90 días para su cancelación, caso contrario deberá reclasificarse.

Obligaciones financieras: comprende las obligaciones contraídas por la empresa a otras instituciones financieras locales o del exterior en calidad de préstamos debido a necesidades de flujo de caja y otros, además la recompra de inversiones. Debido a la naturaleza de este grupo de cuentas generan interés y otros rendimientos financieros que deben ser registrados de forma indistinta. El objetivo primordial radica en la cobertura de préstamos excesivos y retiros no previstos.

Capital social: comprende las aportaciones de los socios en cuento al capital suscrito y pagado más las aportaciones que se registren, que pueden ser en efectivo o mediante el endoso de bienes, el mismo que otorga a los socios derechos proporcionales según su participación.

Resultados: refleja el resultado general de las operaciones efectuadas por la organización en un periodo contable determinado, en donde se puede evidenciar la diferencia de todos sus ingresos contra los gastos realizados para giro mismo del negocio, los cuales se ven representados como pérdidas o ganancias del ejercicio, permitiendo evidenciar el comportamiento de la organización frente al mercado en que se desarrolla.

Cuentas de ingresos: son considerados como aumento del patrimonio, resultados de las actividades económicas generadas diariamente, y pueden provenir de la venta de bienes y demás que generen utilidad para el ente económico. (Alcarria, 2009). Los grupos de cuentas más utilizadas son: intereses y descuentos ganados, intereses por servicios y otros ingresos, que son recaudados lícitamente y a través de las operaciones organizacionales.

Cuentas de Gastos: Fullana y Paredes (2008) mencionan que el gasto efectuado para el normal desarrollo de sus actividades es considerado como una disminución del activo o por consiguiente del patrimonio, debido al flujo de operaciones diarias; que pueden o no volver a incrementar el pasivo. Dentro de este grupo las más utilizadas son: intereses causados, 
provisiones, gastos de operación, impuestos y otros gastos, que inciden en el mejoramiento de la actividad propia del negocio con aras a un crecimiento y posicionamiento en el mercado competitivo.

A partir del conocimientos básico de las cuentas que generalmente son utilizadas por una cooperativa de ahorro y crédito del sector popular y solidario es necesario identificar los instrumentos que se deben utilizar en los procesos de auditoría interna, con la finalidad de realizar análisis, cálculos y constataciones que sustentar la opinión del profesional, los mismos que serán reflejados en los papeles de trabajo debidamente referenciados. En este contexto, a continuación se presentan los principales instrumentos utilizados en la auditoria interna:

Cuestionarios de control interno: constituyen un documento donde el auditor indaga con todos los empleados de la organización, la situación actual con énfasis en priorizar las áreas que presenten alertas para que la auditoría pueda centrarse en su evaluación, los cuestionarios contienen preguntas estratégicas enfocadas en obtener información que revela si la cooperativa mantiene registros exactos y cada una de las personas responsables de ellos.

Papeles de trabajo: los papeles de trabajo según Martinez (2003) son documentos o cédulas elaboradas y diseñadas por el auditor para un proceso de auditoría determinado, en estos papeles se puede evidenciar todos los análisis, procedimientos, cálculos, notas y conclusiones previas que son realizadas durante la ejecución del examen, en su contenido se incluirá una codificación individual, y se detallará marcas de auditoría escritas con esfero de color rojo, y el resto de contenido se lo puede realizar con lápiz, cumpliendo con las condiciones de fondo y forma establecidos por las NAGAS. Para la referenciación se utilizará una codificación o combinación alfa numérica, asignándole a cada papel o cédula una letra mayúscula del alfabeto seguido de un número ordenado cronológicamente, que servirá de guía en el proceso continuo del examen; se podrá conceptualizar cada letra según sea la importancia o incidencia dentro del análisis (Estupiñan, 2013).

Simbología: la elaboración de los papeles de trabajo deberá realizarse de forma ordenada y clara a fin de evitar posibles errores o confusiones, como ayuda se puede referenciar los datos señalando el origen de la información (Vanegas, 2007) además se puede marcar cada acontecimiento con símbolos para que sea de más fácil acceso e interpretación, estos deberán estar determinados preliminarmente o contenido su significado en el pie del papel o cédula, como ejemplo citamos algunas marcas de auditoría:

/ Cálculos

( ) Documentos

Q Cotejado 
Informe de auditoría: el informe de auditoría es el resultado final del trabajo efectuado por el auditor, en donde se plasman todas las conclusiones del examen, contendrá de forma esencial lo siguiente:

(a) Veredicto del auditor encargado del examen.

(b) Balance general.

(c) Estado de resultado y cambios en el patrimonio.

(d) Estado de Flujo del efectivo.

(e) Notas a los estados y papeles de trabajo.

El auditor luego del proceso de auditoría podrá emitir su opinión sobre los estados financieros de manera favorable (sin salvedad) o negativamente (con salvedad), y como un privilegio podría abstenerse cuando no cuenta con toda la información necesaria dentro del examen.

\section{Metodologia.}

El presente estudio se elaboró bajo un diseño no experimental, ya que no se manipularon deliberadamente las variables, en tal razón, el aseguramiento de la información financiera y la auditoría interna fueron analizadas en la unidad de análisis bajo su contexto natural.

El enfoque de la investigación fue cuali - cuantitativo, presentando mayor predominio en el aspecto cualitativo mediante la revisión de la literatura especializada en auditoría financiera e información contable financiera, para la posterior elaboración del marco teórico. Con menor incidencia se aplicó el enfoque cuantitativo mediante el estudio de la población y representación gráfica de la información obtenida a través de datos numéricos procesados en los programas informáticos SPSS versión 23 y Microsoft Excel 2013. El enfoque fue de carácter descriptivo / explicativo dado que en la fase de estructuración del marco teórico se pormenorizó el proceso completo de auditoría financiera y la descripción del proceso de aseguramiento de la información contable y financiera, de manera conjunta se transformó en explicativo debido a que en la elaboración de la propuesta se fue explicando y detallando cada uno de los componentes y pasos a seguir dentro del procedimiento metodológico de auditoría interna.

Los métodos utilizados fueron: el histórico - lógico, empleado en el análisis de la trayectoria de la auditoria y de las normas y leyes generales que rigen su funcionamiento. El inductivo - deductivo, mediante la implementación de estrategias de razonamiento lógico permitió establecer premisas particulares de las variables de estudio para llegar a conclusiones generales, así como también basado en principios generales se logró establecer conclusiones específicas sobre la problemática indagada en la unidad de análisis. El método analítico sintético permitió la descomposición de las variables de estudio con la finalidad de analizar 
detenidamente cada una de sus elementos y posteriormente bajo un proceso de razonamiento lógico se reconstruyeron las teorías integrando las partes del todo.

Las técnicas que se emplearon en el estudio fueron: la entrevista dirigida al gerente de la cooperativa de ahorro y crédito Santiago Ltda; de la ciudad de Loja, Ecuador, la encuesta que se dirigió a la Junta General de Representantes (30), integrantes del Consejo de Administración (5), integrantes del Consejo de Vigilancia (3) y Contadora. Por lo antes expuesto, la población de estudio estuvo conformado por 40 personas.

\section{Resultados.}

Los resultados obtenidos de la aplicación de los instrumentos de investigación que sirvieron para el análisis del control que se establece dentro de la cooperativa de ahorro y crédito Santiago Ltda., para el aseguramiento de la información contable financiera, se plasman a continuación:

\section{Estado de situación financiera.}

En el estado de situación financiera se demuestra el resultado financiero de una organización dentro de un determinado periodo, este estado permite realizar comparaciones para una acertada toma de decisiones. Se estructura de las cuentas de activo, pasivo y patrimonio las cuales mediante un proceso integro dentro de su tratamiento denotan sus resultados.

En este sentido, se determinó que en la Cooperativa de Ahorro y Crédito Santiago Ltda; la información relaciona al estado de situación financiera y cada una de las cuentas que lo conforman no cuentan con procedimientos aprobados para su manejo y aplicación, lo que ocasiona que existan falencias dentro del cumplimiento de las disposiciones legales, con esto la información financiera presentada carezca de claridad y veracidad.

\section{Estado de Resultados.}

Este estado presenta de forma ordenada y detallada cada uno de los rubros que intervino para la determinación del resultado económico en un periodo fiscal, dentro de este estado se detallan los costos y gastos que fijaron los ingresos del periodo para poder determinar el resultado positivo o negativo para la organización antes y después de impuestos previstos por la Ley.

Del diagnóstico efectuado sobre este indicador se determinó que la Cooperativa cuenta con los respaldos de los ingresos registrados en su sistema contable, sin embargo, no se evidencio procedimientos de control interno. 


\section{Estado de flujo del efectivo.}

El estado de flujo de efectivo demuestra las variaciones y movimiento de dinero que se dieron al interno de la organización resultante de las operaciones efectuadas, refleja además el total de efectivo que se generó y su aprovechamiento como tal, demostrándose el origen de mayor relevancia del efectivo, su destino y su producción generadora.

La cooperativa realiza actividades de intermediación financiera lo que ocasiona que la mayor fuente de ingresos y mayor movimiento de efectivo provenga de esta actividad, diferenciándose los tipos de flujo de efectivo de acuerdo a sus operaciones realizadas diariamente, sin embargo, se evidencia que existe una falta de categorización de cada una de las cuentas generadoras de ingresos.

Los ingresos generados por las actividades de intermediación financiera como depósitos, retiros, préstamos, etc.; no se diferencian de los ingresos provenientes de las actividades análogas a la cooperativa como servicios anexos (Pago de luz, etc.) por lo que ocasiona que no se realice una interpretación adecuada de este indicador afectando directamente a la toma de decisiones.

\section{Notas a los estados financieros.}

Representan las aclaraciones o explicaciones de hechos o circunstancias que están relacionadas al movimiento de las cuentas intervinientes en cada estado financiero, lo que aporta a un entendimiento claro y razonado de cada indicador.

Las notas aclaratorias presentadas en los estados financiero de la Cooperativa de Ahorro y Crédito Santiago Ltda; no revelan toda la información representativa que ha incidido significativamente dentro en la elaboración de la información financiera, por consiguiente se desconoce los cálculos, técnicas y métodos empleados.

\section{Presupuesto Institucional.}

El presupuesto de una cooperativa de ahorro y crédito está determinado como el límite de gastos que se deben realizar para poder conseguir los objetivos planteados así como el detalle de los ingresos que cubrirán estos gastos dentro un perdió de tiempo determinado, es necesario que se realice controles de cumplimento a la ejecución del mismo así como la documentación que respalde las actividades desarrolladas y aprobadas.

En la Cooperativa de ahorro y crédito Santiago Ltda; se estableció que para la formulación del presupuesto institucional se lo realiza en base a experiencias de periodos anteriores y no en base a los planes y metas trazadas para el periodo vigente. 


\section{Auditoría Interna.}

La auditoría interna es un proceso de control que se realiza a las actividades diarias con énfasis a la verificación del cumplimiento íntegro de las normas, políticas y procedimientos establecidos interna como externamente, todo esto para proteger sus activos y poder minimizar el riesgo presente en cada una de sus operaciones.

Con esta premisa, se pudo determinar que la Cooperativa no cuenta con un departamento de auditoría interna, en tal razón, la evaluación de los estados financieros se lo hace únicamente a través de auditorías externas.

A partir de estos resultados, se deriva la necesidad de establecer un procedimiento metodológico de auditoría interna con la finalidad de mejorar los procesos de control sobre la información financiera, la gestión administrativa y operativa de la Cooperativa de Ahorro y Crédito Santiago Ltda. El procedimiento metodológico está estructurado por las fases de planificación, ejecución e informe de auditoría, lo que permite lograr el desarrollo óptimo y eficiente del trabajo de auditoria con la finalidad de contar con información confiable y oportuna (Figura 1).

Figura 1. Esquema de la propuesta del proceso metodólogo de auditoría interna.

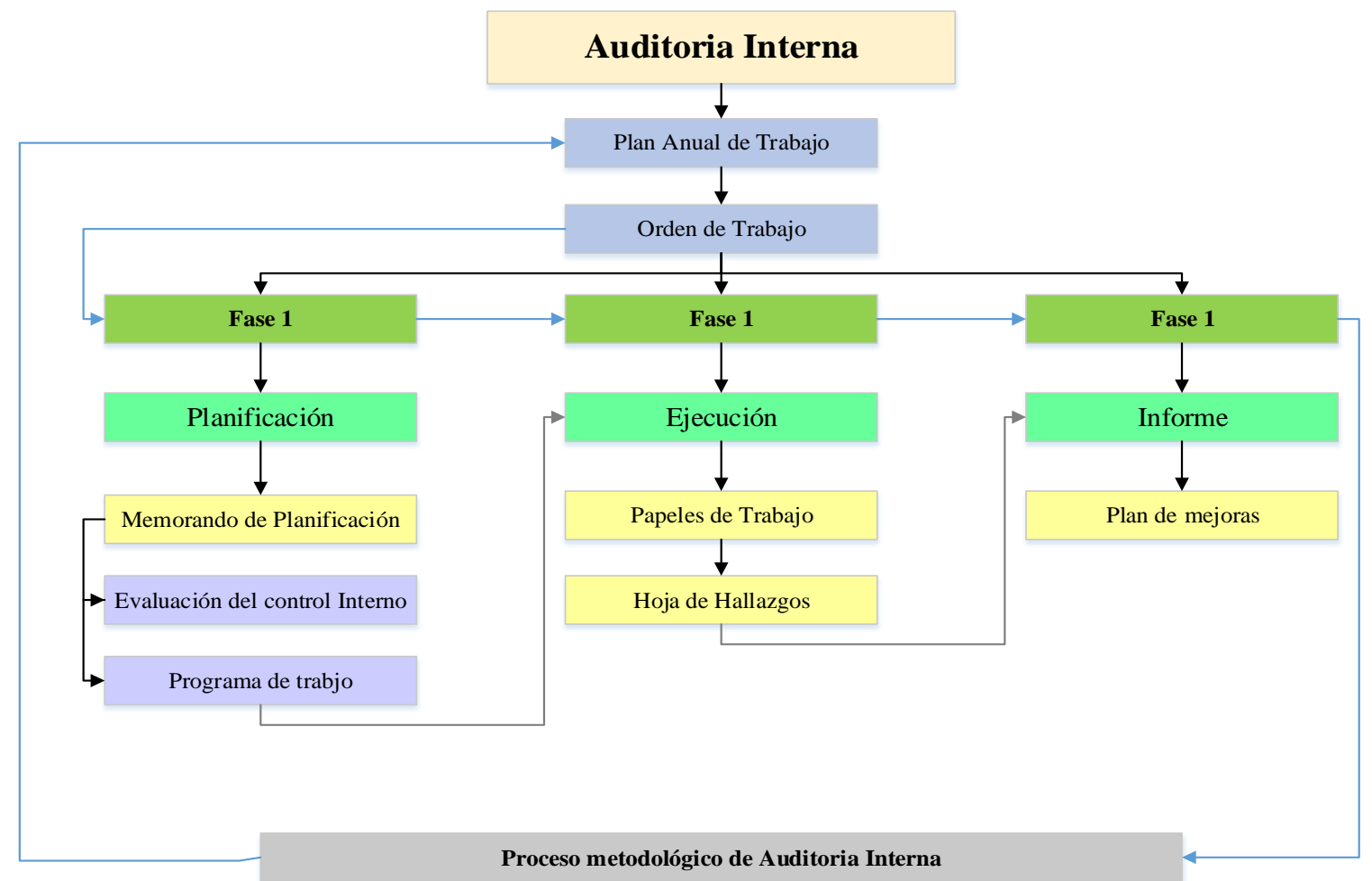


La auditoría interna en el sector financiero popular y solidario inicia con la elaboración del plan de trabajo de Auditoria Interna, dentro del cual se detallan todas las actividades que el auditor interno debe ejecutar en un periodo fiscal, tomando en consideración las normativas vigentes emitidas por los organismos de control y las normativas propias de la Cooperativa de Ahorro y Crédito Santiago Ltda. En la tabla 02 se describe el procedimiento correspondiente.

Tabla 2. Plan de Trabajo de Auditoria Interna.

\section{Procedimiento Metodológico}

Nombre: Plan de Trabajo de Auditoria Interna.

Objetivo: Identificar las actividades que se van a desarrollar para el control objetivo de sus actividades durante el ejercicio contable.

\section{Procedimiento:}

1. Describir los datos generales del Plan de trabajo como: fecha de inicio, fecha de finalización, fecha de aprobación y datos del Auditor Interno.

2. Describir una introducción que describa brevemente la naturaleza de las áreas a ser evaluadas y resumen los procedimientos realizadas diariamente.

3. Objetivo que persigue el plan de trabajo al realizar la evaluación de las actividades.

4. Definir el Plan de Trabajo mediante la selección de la normativa legal, actividades a realizar en cada componente de la cooperativa, así como los documentos soporte que deben ser entregados, periodicidad de aplicación y los plazos estimados para su ejecución.

5. Se debe realizar controles de fechas para no incurrir en feriados, ni que el plan de trabajo sobrepase el año fiscal.

Responsables: Auditor Interno y Presidente del Consejo de vigilancia

Entregables: Plan de trabajo de Auditoria Interna.

Cabe recalcar que el Auditor Interno debe estar debidamente designado por la Cooperativa de Ahorro y Crédito y puesto a consideración de la Superentendía en un término máximo de 15 días a partir de su designación, y su trabajo se regirá al Código de Ética Profesional que rescata los principios de: Integridad, objetividad, confidencialidad y competencia. Para la ejecución del plan de trabajo es necesario que el Consejo de Vigilancia de la Cooperativa solicite al Gerente una orden de trabajo, al auditor interno para que realice un proceso de control y dentro de la cual se describen el trabajo a ejecutar, en la Tabla 03 se describe el procedimiento para su estructuración.

Tabla 3. Proceso metodológica de Orden de Trabajo.

\footnotetext{
Procedimiento Metodológico

Nombre: Orden de trabajo.

Objetivo: Informar detalladamente el procedimiento que se deberá aplicar el desarrollo de la auditoria interna

Procedimiento: la orden de trabajo es un oficio donde se da a conocer sobre la aplicación de la auditoria y se estructura de la siguiente manera:
} 
1. Lugar y fecha.

2. Nombre y cargo a quien va direccionado.

3. Contexto en donde se da a conocer: objetivo, alcance, tiempos estimados de ejecución e instrucciones generales para la aplicación de auditoria.

4. Firma de responsabilidad.

5. Se deberá soportar con la fe de recepción.

Responsables: Presidente del Consejo de vigilancia y Gerente

Entregables: Orden de trabajo

Con esta orden de trabajo el Auditor Interno debe desarrollar una serie de actividades para dar estricto cumplimiento al objetivo presentado dentro de esta orden, por lo cual y en base a la normativa legal vigente el proceso de auditoría interna debe contener las siguientes fases:

La primera fase de auditoria y de su juicio dependerá el resultado del trabajo ejecutado, el objetivo primordial de la planificación es obtener y restablecer información de la Cooperativa y sus actividades diarias, dentro de esta fase se debe de considerar alternativas y condensar métodos que ayuden a la ejecución plena de las actividades de control. Esta fase inicia con el conocimiento de la cooperativa y finaliza con la determinación de las tareas a realizarse.

Para la planificación de auditoría interna es necesario desarrollar un memorando de Planificación, que es un resumen sistemático del desarrollo de toda la planificación que sirve para dar a conocer de manera discreta la etapa de familiarización, evaluación del control interno, y las actividades a desarrollarse en la fase de ejecución, en la tabla 04, se describe el procedimiento para la elaboración del mismo.

Tabla 4. Proceso metodológico para memorando de Planificación

\section{Procedimiento Metodológico}

Nombre: Memorando de planificación.

Objetivo: Dar a conocer los resultados obtenidos del conocimiento previo de la cooperativa.

Procedimiento:

1. Se presenta una recapitulación de la auditoria que contiene: antecedentes, motivo, objetivos y alcance.

2. Realizar una presentación general de la Cooperativa: normativa legal, políticas y principales actividades desarrolladas, sistemas contables y grado de confiabilidad de la información.

3. Se deben definir puntos de interés para ser evaluados, convirtiéndose en recursos analizados.

4. Servidores relacionados directamente con la manipulación de información objeto de interés.

5. Detallar los componentes y sub componentes priorizados y que requieren un análisis minucioso.

6. En caso de requerirse se deberá aplicar técnicas de muestreo para poder analizar la información pertinente.

7. Evaluar el control interno mediante la aplicación de cuestionarios.

8. Desarrollar los programa de auditoria basados en la calificación del riesgo.

9. Adicionalmente se deberá describir los recursos a utilizar, el tiempo necesario para cada componente, las marcas de auditoria y los productos esperados del resultado del análisis. 
10. De existir se debe analizar el seguimiento a recomendaciones de exámenes anteriores.

11. Estructurar una matriz de evaluación preliminar del riesgo.

12. Definir actividades sustantivas que deberán ser evaluadas en la siguiente fase.

Responsables: Auditor Interno

Entregables: Memorando de planificación.

Para el desarrollo del memorando de planificación es necesario la aplicación de métodos que permitan evaluar el control interno de cada una de las áreas de la cooperativa, uno de los métodos más esenciales y reveladores es la aplicación de cuestionarios puesto que nos permiten conocer con gran certeza las áreas de mayor focalización de riesgos que tengan incidencia directa con el desarrollo normal de la cooperativa, en la tabla 05 se describe el proceso metodológico para la elaboración de los cuestionarios.

Tabla 5. Proceso metodológico para cuestionario de control interno.

\section{Procedimiento Metodológico}

Nombre: Cuestionario de control interno.

Objetivo: Reunir información, para describir hechos u opiniones de posibles hallazgos.

Procedimiento:

1. Encabezado. Contiene los datos de identificación de la cooperativa, área y rubro examinado, nombre específico del procedimiento y referencias necesarias.

2. Cuerpo: dentro del cuerpo del cuestionario debe contener preguntas enfocadas a obtener un conocimiento del manejo de las operaciones, de las personas involucradas y controles efectuados de acuerdo a cada área.

3. Se debe tener en consideración lo siguiente para la formulación del cuestionario:

- Se debe redactar a nivel de formación del encuestado.

- Las preguntas deben ser claras, precisas y sin demostrar inclinación a una determinada respuesta.

- Debe evitarse los términos subjetivos.

- Las preguntas no deben ser largas, a fin de evitar la fatiga.

- Las preguntas deben estar inclinadas a ayudar al logro de los objetivos.

4. Se debe poner una calificación ponderada a cada pregunta, para poder determinar el nivel de confiabilidad y riesgo.

5. Pie. En la parte final del cuestionario se debe evidenciar por quien fue elaborado y quien reviso este cuestionario.

Responsable: Auditor Interno

Entregables: Cuestionarios de control interno.

Con los resultados obtenidos de los cuestionarios de control interno y su calificación del riesgo se estructura los programas de trabajo de auditoria, los mismos que tienen como objetivo principal describir de manera eficiente los procesos que el auditor interno realizara en aras de obtener evidencia suficiente y relevante de las actividades desarrolladas, con los elaboración de los programas se materializa la planificación, en la Tabla 06 se detalla el procedimientos metodológico de estructuración de un programa de trabajo. 
Tabla 6. Proceso metodológico para programa de Auditoria Interna.

\section{Procedimiento Metodológico}

Nombre: Programa de Auditoria.

Objetivo: Describir los procedimientos que sirven como guía y control del trabajo.

Procedimiento:

1. Encabezado. Contiene los datos de identificación de la cooperativa, periodo, nombre específico del procedimiento y referencias necesarias.

2. Debe contener el objetivo principal de auditoria que se pretende alcanzar con la ejecución de este procedimiento.

3. Cuerpo: dentro del cuerpo del cuestionario debe contener las actividades a realizar basados en los controles efectuados de cada área, se considerar lo siguiente para su formulación:

- Las actividades deben describir plenamente lo que se tiene que realizar y lo que se va a conseguir.

- Debe evitarse los términos subjetivos.

- Las actividades deben ayudar al logro de los objetivos.

4. Se debe referenciar los papeles de trabajo q fuesen necesarios para concluir con la actividad descrita, así como observaciones que ayuden a clarificar las conclusiones.

5. Pie. En la parte final del cuestionario se debe evidenciar por quien fue elaborado y quien reviso este cuestionario como las fechas en las que se realizó.

Responsable: Auditor Interno.

Entregables: Programa de auditoria.

A más de existir normativas legales para el uso y manejo de la información financiera existen varias disposiciones legales que debe cumplir la cooperativa debido al manejo de dinero diario que por su actividad se genera y puesto esto se facilita para el cometimiento de ilícitos, la Unidad de Análisis Financiero (UAF) solicita de manera periódica información sobre las operaciones inusuales. En la Tabla 7 se presentan el programa de trabajo para demostrar el cumplimento de la normativa establecida por la UAF.

Tabla 7. Programa de trabajo para la unidad de análisis financiero

\begin{tabular}{|c|c|c|c|c|c|}
\hline \multicolumn{6}{|c|}{$\begin{array}{c}\text { Cooperativa de Ahorro y Crédito Santiago Ltda. } \\
\text { Programa de Trabajo } \\
\text { Unidad de analisis financiero } \\
\text { Periodo del al }\end{array}$} \\
\hline \multicolumn{6}{|c|}{$\begin{array}{l}\text { Objetivo: Revisar los procedimientos y políticas implantadas para contrarrestar el lavado de activos y } \\
\text { operaciones inusuales. }\end{array}$} \\
\hline \# & Procedimiento & Ref/p.t & Realizado & Revisado & Observaciones \\
\hline 1 & $\begin{array}{l}\text { Obtener información personal sobre el oficial de } \\
\text { cumplimento. }\end{array}$ & & & & \\
\hline 2 & $\begin{array}{l}\text { Verificar si el oficial de cumplimiento designado } \\
\text { cumple con todos los requisitos de ley y está } \\
\text { acreditado por la SEPS. }\end{array}$ & & & & \\
\hline
\end{tabular}




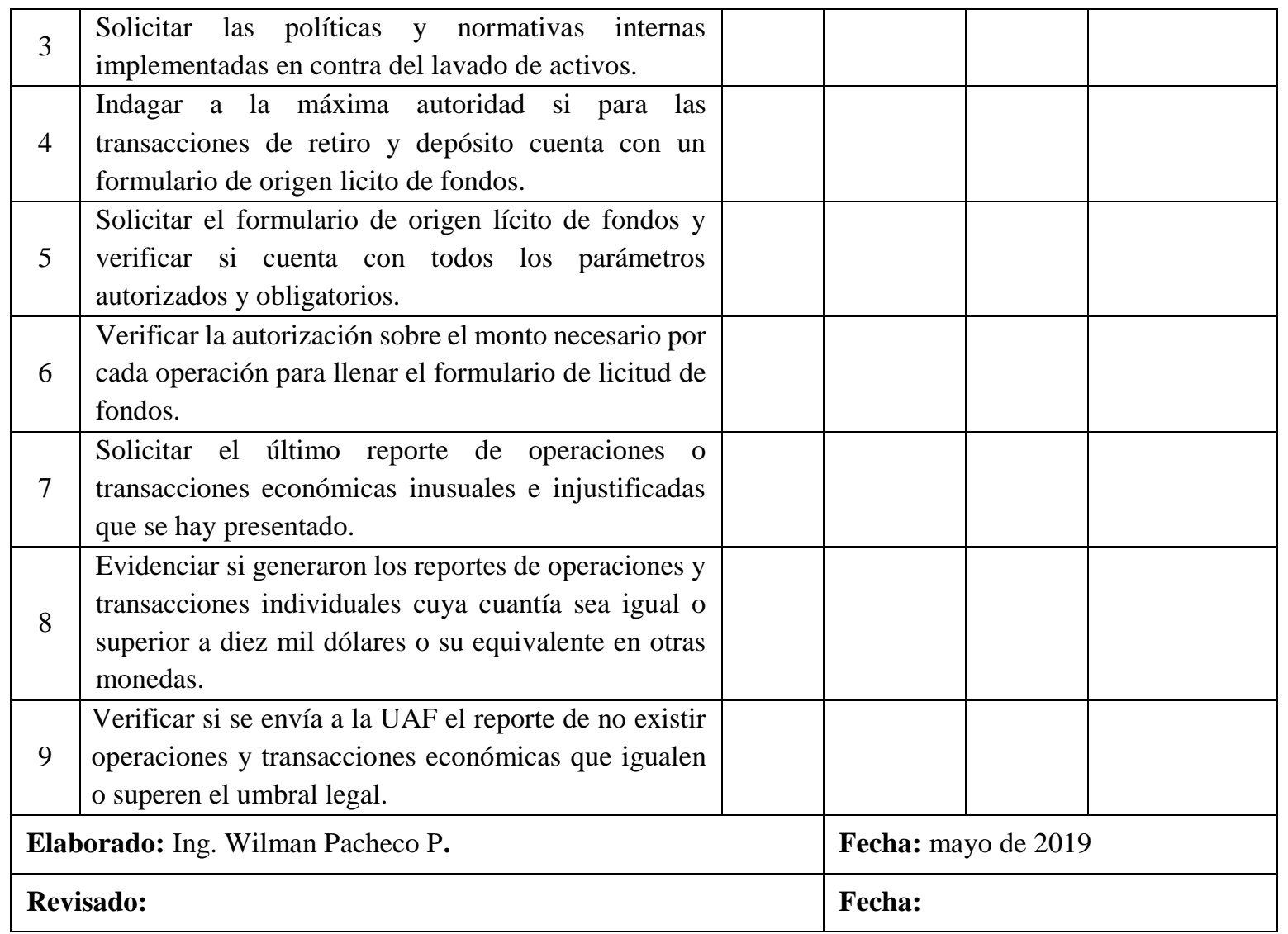

En el ámbito tributario y por las actividades de intermediación financiera que se realizan en la cooperativa, están obligados a cumplir obligaciones establecidos guiándose en leyes, reglamentos, normas y procedimientos que se encuentran establecidas por el organismo de control que es el Servicio de Rentas Internas. En la tabla 8 se presenta la revisión necesaria para evidenciar el cumplimento tributario.

Tabla 8. Programa de trabajo para la revisión del cumplimento tributario.

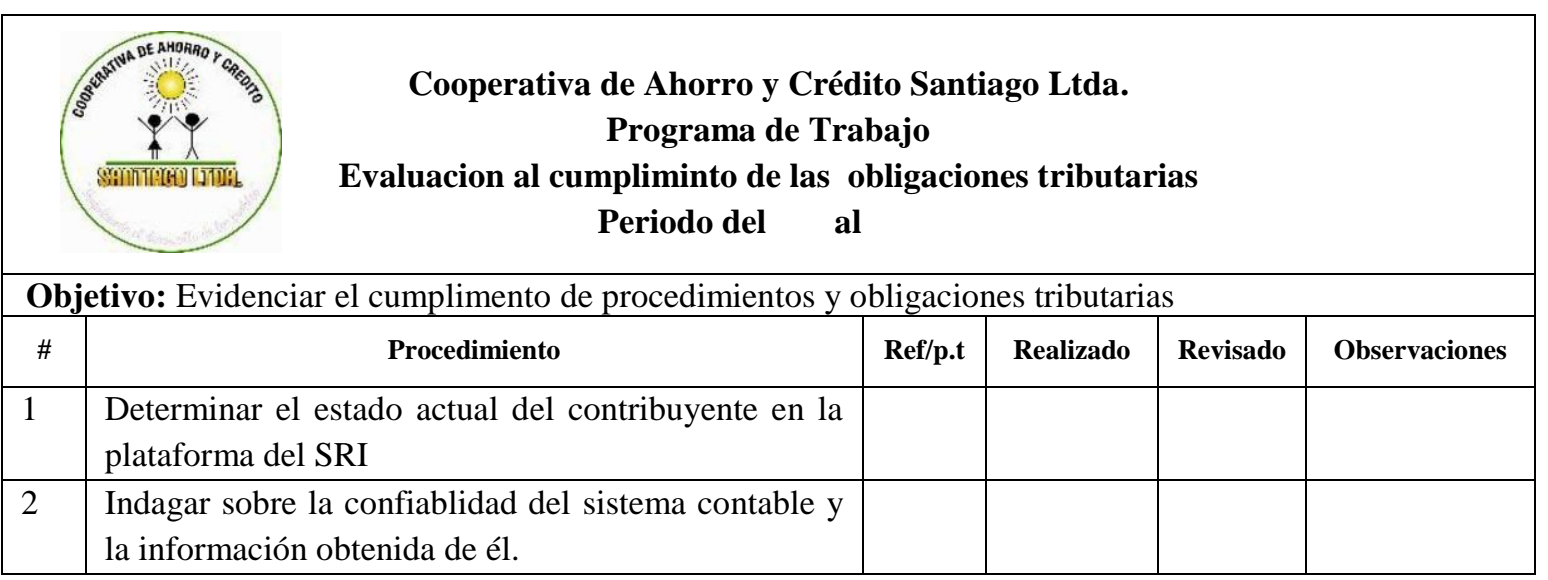




\begin{tabular}{|c|c|c|c|}
\hline 3 & $\begin{array}{l}\text { Solicitar auxiliares de la cuenta de impuestos } \\
\text { causados. }\end{array}$ & & \\
\hline 4 & $\begin{array}{l}\text { Solicitar los formularios de declaración del impuesto } \\
\text { a la renta anual y verificar la información contenida. }\end{array}$ & & \\
\hline 5 & $\begin{array}{l}\text { Solicitar las declaraciones mensuales realizadas del } \\
\text { IVA e impuesto a la renta }\end{array}$ & & \\
\hline 6 & $\begin{array}{l}\text { Contrastar los valores pagados al SRI vs valores } \\
\text { contables. }\end{array}$ & & \\
\hline 7 & $\begin{array}{l}\text { Indagar si dentro del periodo contable se han } \\
\text { realizado retenciones. }\end{array}$ & & \\
\hline 8 & $\begin{array}{l}\text { Evidenciar si dentro de las declaraciones efectuadas } \\
\text { consta el valor exacto d las declaraciones. }\end{array}$ & & \\
\hline 9 & $\begin{array}{l}\text { Evidenciar si se realizaron las declaraciones } \\
\text { tributarias por motivos de vehículos y propiedades. }\end{array}$ & & \\
\hline 10 & $\begin{array}{l}\text { Calcular el total de multas e interés causados por no } \\
\text { realizar las declaraciones oportunamente. }\end{array}$ & & \\
\hline 11 & $\begin{array}{l}\text { Evidenciar la secuencia de los comprobantes } \\
\text { autorizados por el SRI. }\end{array}$ & & \\
\hline 12 & $\begin{array}{l}\text { Solicitar el ultimo anexo de relación de dependencia } \\
\text { presentado }\end{array}$ & & \\
\hline \multicolumn{2}{|c|}{ Elaborado: Ing. Wilman Pacheco P. } & \multicolumn{2}{|l|}{ Fecha: mayo de 2019} \\
\hline \multicolumn{2}{|c|}{ Revisado: } & \multicolumn{2}{|l|}{ Fecha: } \\
\hline
\end{tabular}

Por su parte la SEPS como organismo rector de las cooperativas establece que dentro de su ámbito de incidencia debe estar considera la responsabilidad social que tienen con sus socios, grupos de interés y medio ambiente en general, por tal razón se debe presentador estos indicadores mediante un balance social cooperativista. La tabla 9 demuestra el programa de trabajo para evidenciar el cumplimento de los principios y objetivos sociales.

Tabla 9. Programa de trabajo para la unidad de análisis financiero

\begin{tabular}{|l} 
Cooperativa de Ahorro y Crédito Santiago Ltda. \\
Programa de Trabajo \\
Evaluacion del Balance Social \\
al \\
Periodo del
\end{tabular}




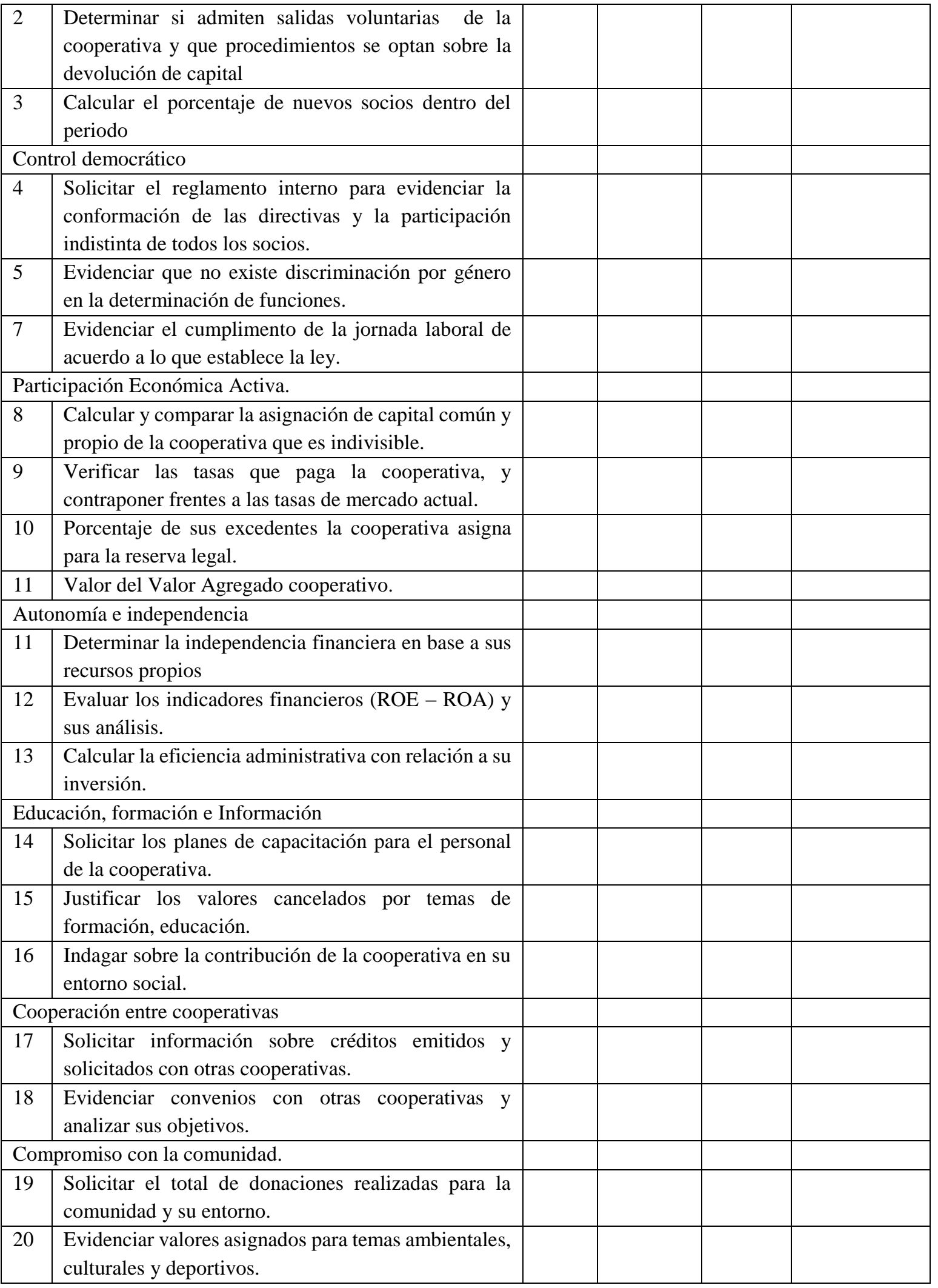




\begin{tabular}{|l|l|}
\hline Elaborado: Ing. Wilman Pacheco P. & Fecha: Mayo de 2019 \\
\hline Revisado: & Fecha: \\
\hline
\end{tabular}

La segunda fase de la auditoria da inicio con la aplicación de los programas detallados en la fase de planificación con la finalidad de obtener información suficiente y competente para poder demostrar o sustentar las afirmaciones o aseveraciones realizadas, durante esta etapa se realizan pruebas a través de técnicas de auditoria a los diferentes procesos de información en cuanto a su razonabilidad y veracidad, para ello es necesario realizar papeles de trabajo en el cual se detallan cada uno de los procedimientos empleados en la evaluación, estos documentos solidifican la determinación de hallazgos que son presentados en una hoja de hallazgos o también conocida como cedula narrativa.

Para la elaboración de los papeles de trabajo es necesario contar con la información relacionada a las actividades priorizadas y catalogadas como riesgosas, por lo cual de forma escrita a los servidores relacionados se solicita la información necesaria y pertinente que debe estar certificada y legalizada, en su análisis se generan cálculos, procedimiento necesarios para poder comprobar su veracidad, todos estos procedimiento se deben plasmar en los papeles de trabajo.

Los papeles de trabajo no mantienen un formato estandarizado si no por el contrario se adaptan a la necesidad y entendimiento del profesional de auditoria. En la Tabla 10 se presenta el procedimiento metodológico para la elaboración de un formato base.

Tabla 10. Proceso metodológico para papeles de Trabajo

\section{Procedimiento Metodológico}

Nombre: Papeles de Trabajo.

Objetivo: Plasmar los procedimientos, cálculos y observaciones encontradas dentro de la evaluación de los rubros objeto de auditoria.

\section{Procedimiento:}

1. Encabezado. Contiene los datos de identificación de la cooperativa, área y rubro examinado, nombre específico del procedimiento.

2. Clave o índice del papel de trabajo, iniciales de quien realizo el análisis y la fecha en que fue realizado.

3. Cuerpo del papel de trabajo que contiene la evidencia recolectada objeto del análisis, las pruebas realizadas y los resultados obtenidos en base a la verificación de normativas legales pertinentes básicamente contiene: cantidades y conceptos revisados, referencias, observaciones de irregularidades, conclusiones parciales y marcas de auditoria

4. En el pie del papel de trabajo se encuentra: notas aclaratorias, significado de las marcas de auditoria utilizadas, fuente de recolección y conclusión definitiva

5. Finalmente se debe plasmar por quien fue elaborado y en qué fecha

Responsable: Auditor Interno

Entregables: Papeles de trabajo 
En la hoja de hallazgos o cedulas narrativas se detallan las debilidades encontradas en la aplicación del control interno, y contienen la información que debe ser puesta a consideración de las autoridades de la cooperativa y de las personas comprometidas en este hallazgo, puesto con esta información se da a conocer las deficiencias importantes que podrán inferir negativamente al normal desarrollo de las actividades, en la tabla 11 se muestra el proceso metodológico de elaboración de una hoja de hallazgos.

Tabla 11. Proceso metodológico para Hoja de Hallazgos

\section{Procedimiento Metodológico}

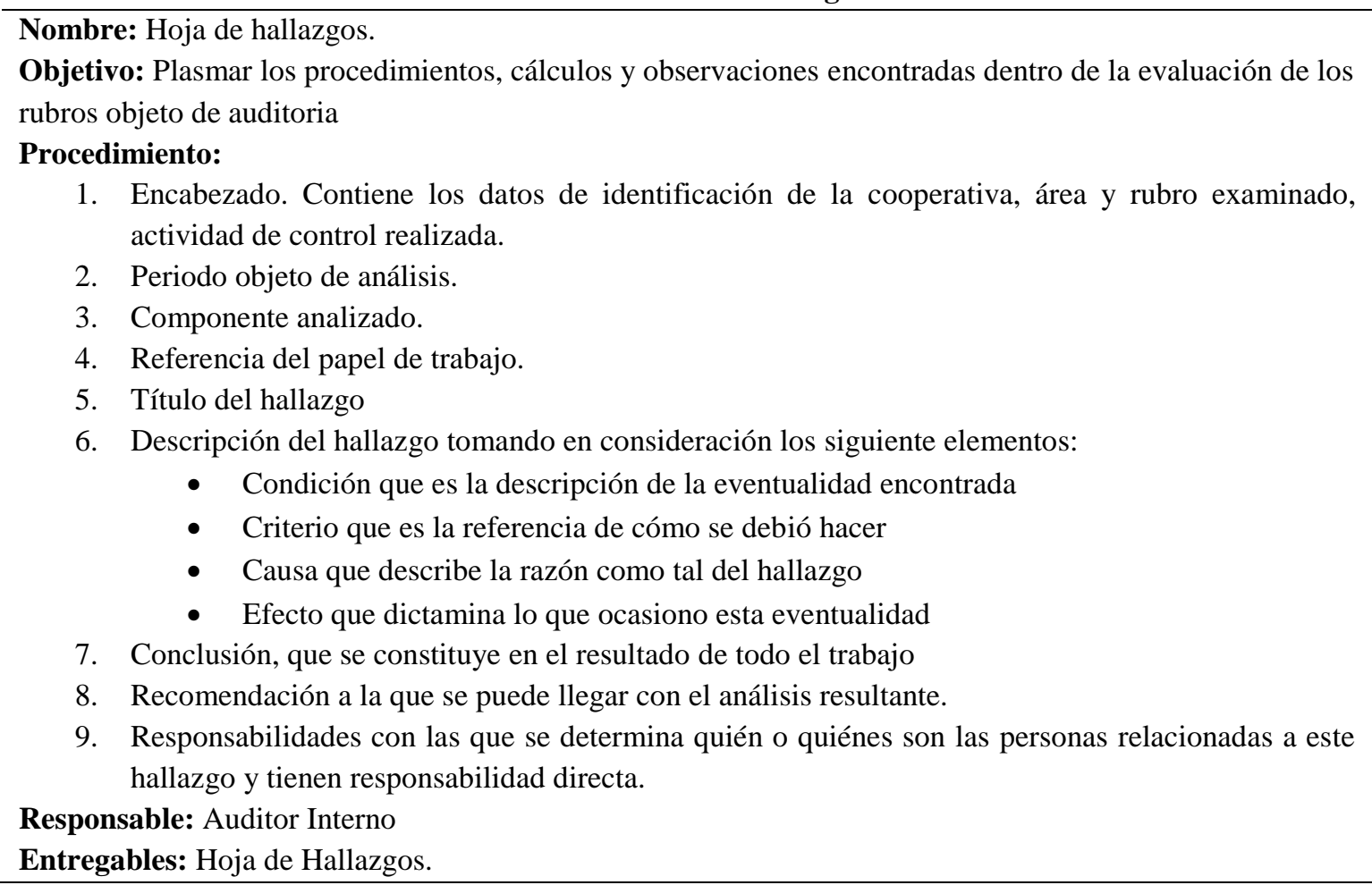

Una vez concluido con la aplicación de los programas de auditoria y obtenido los hallazgos necesarios para fundamentar las opiniones previas es necesario formalizar los resultados obtenidos, lo que se realiza mediante la presentación del informe preliminar de resultados que demuestra detalladamente lo que fue examinado matizando puntos positivos y negativos y la conclusión a la que se pudo llegar con esta evaluación, informando a la administración de la cooperativa de la situación actual de la misma.

Previa a la elaboración del informe se debe realizar una recopilación de toda la información generada en el transcurso de la ejecución de auditoria, con la finalidad de realizar una lectura y análisis que pueda afianzar las conclusiones y recomendaciones, es recomendable realizar un resumen de toda el proceso lo que facilitara la elaboración del informe de auditoría que 
será presentado al Gerente y los Órganos directivos de la cooperativa, Para la elaboración del informe se debe de considerar lo siguiente:

- $\quad$ Debe ser claro, preciso, completo y de fácil entendimiento.

- $\quad$ Presentar los resultados de manera objetiva y documentada.

- Redactar libre de palabras antagónicas.

- Relacionar los hallazgos positivos dentro del informe.

- Buscar culpables o determinar quién fallo

En la Tabla 12 se describe el procedimiento metodológico para elaborar el informe de auditoría.

Tabla 12. Proceso metodológico para la elaboración del informe de auditoría Interna Procedimiento Metodológico

Nombre: Informe de auditoría.

Objetivo: Plasmar los procedimientos, cálculos y observaciones encontradas dentro de la evaluación de los rubros objeto de auditoria.

Procedimiento: El informe se estructura de 2 capítulos:

Capítulo 1.

1. Información introductoria del examen que contiene: motivo, objetivos y alcance.

2. Resumen del conocimiento de la cooperativa: base legal, estructura orgánica y objetivos.

3. Rubros o áreas examinados, que es donde se detalla a que rubro o a que área de la cooperativa se aplicó el examen.

4. Colaboradores relacionados, se describe quien está directamente relacionado con la elaboración o manipulación de la información.

Capítulo 2.

1. Seguimiento a recomendaciones. Que es evidenciar si se dio cumplimiento a las recomendaciones emitidas en evaluaciones anteriores.

2. Dar a conocer los hallazgos encontrados y para ellos se presentara en base al siguiente esquema: título del hallazgo, conclusión a la que se pudo llegar y recomendaciones para mejorar la condición.

3. Firma de responsabilidad del auditor.

Responsable: Auditor interno

Entregables: Informe final de Auditoria.

La comunicación de resultados es en base al informe de auditoría en la cual mediante una socialización con las principales autoridades de la cooperativa y personas vinculadas en el examen, se da a conocer a nivel macro el estudio realizado dando a conocer las debilidades y mejoras que se pueden dar al sistema de control interno.

Para finalizar con el proceso el Auditor Interno realizara la entrega al Gerente y Consejo de Vigilancia un plan de recomendaciones o mejoras planteadas que tiene como propósito fundamental plantear actividades como alternativas de solución para mejorar el beneficio de la cooperativa en base a obtener una información real y oportuna, con los hallazgos 
encontrados y en base a las recomendaciones realizadas se deben analizar y plantear actividades, procedimientos o manuales que estén enfocados mitigar el riesgo y asegurar eficientemente la información financiera de la cooperativa, puesto que la misma sirve de punto de partida para una planificación estratégica. En la tabla 13 se describe el procedimiento metodológico que se sigue para estructurar un plan de mejoras aplicable a la cooperativa de ahorro y crédito.

Tabla 13. Proceso metodológico para elaborar un Plan de Mejoras

\section{Procedimiento Metodológico}

Nombre: Informe de auditoría.

Objetivo: Desarrollar una matriz tipo para plantear alternativas de solución a los hallazgos encontrados.

Procedimiento: Se debe elaborar una matriz tipo para plantear actividades enfocadas a solucionar los riesgos encontrados, de lo cual debe contener:

1. Riesgos / Hallazgos: Se plasman los hallazgos encontrados en el examen realizado

2. Recomendaciones: son las recomendaciones emitidas por el auditor en el informe de auditoría.

3. Metas: contiene las situaciones de cambio planteadas que se espera alcanzar.

4. Estrategias: son acciones que ayudan a llegar a la meta propuesta, con tiempos determinados. Es necesario conocer el cómo, por qué y para que ejecutarlas

5. Recursos: se refiere a la necesidad de recursos humanos, físicos, financieros y tecnológicos.

6. Fecha: es el tiempo estimado para la aplicación de las estrategias en aras de obtener la meta trazada.

7. Responsable: es el actor quien implementa las estrategias, además de definir participantes y responsabilidades.

8. Seguimiento y evaluación: Permite realizar ajusten de ser necesario en la implementación de la estrategia.

9. Responsable del seguimiento.

10. Estado actual: evidencia las situaciones de cambio.

Responsable: Auditor interno

Entregables: Matriz de plan de mejoras.

Para el fiel cumplimiento del Plan de mejoras el Gerente y Consejo de Vigilancia deberán realizar un seguimiento y control frecuente del cumplimiento de las recomendaciones contendías.

\section{Conclusiones.}

- La información financiera de una organización del sector popular y solidario es de transcendental importancia para su crecimiento competitivo y económico, por consiguiente, con una acertada evaluación se puede tomar decisiones correctas que encaminen a la organización a un crecimiento de su patrimonio.

- El proceso de auditoría interna tiene por objetivo examinar y evaluar la eficaz aplicación de los procesos de control interno, a efectos de velar por la preservación e 
integridad del patrimonio de una organización, así como la eficiencia de su gestión económica.

- La Cooperativa de Ahorro y Crédito Santiago Ltda no cuenta formalmente con procedimientos de control interno para cada una de sus operaciones, en tal razón, no se puede determinar de forma apropiada la efectividad operativa de la institución.

- La propuesta del proceso metodológico de auditoria interna constituye un instrumento eficiente para el control adecuado de los procesos realizados por la cooperativa, por consiguiente, a partir de su aplicación se dará seguridad a la dirección y podrán tomar acciones correctivas respecto a los controles establecidos, a los registros contables, planes políticas y procedimientos empleados para la elaboración de información contable-financiera.

\section{Referencias bibliográficas.}

Alcarria, J. (2009). Contabilidad Financiera 1 (Primera Edicion ed.). España: Universidad Jaume. Obtenido de https://books.google.com.ec/books?id=6m42LTDkhzoC\&pg=PA29\&dq=definici\% $\mathrm{C} 3 \% \mathrm{~B} 3 \mathrm{n}+\mathrm{de}+\mathrm{gastos}+\mathrm{contables} \& \mathrm{hl}=\mathrm{es} \& \mathrm{sa}=\mathrm{X} \& \mathrm{ved}=0 \mathrm{ahUKEwiylczMpaTaAhXKT}$ 8KHZCACjYQ6AEIJjAA\#v=onepage \&q\&f=false

Asamblea, C. (2008). Constitución de la República del Ecuador. Ciudad Alfaro.

Bravo, M. (2011). Contabilidad General. Quito: Nuevodia.

Cardozo, H. (2016). Auditoria delsector solidario. Bogota: ECOE Ediciones.

Catácora, F. (1996). Sistemas y procedimientos contables. Venezuela: McGraw/Hill.

Estupiñan, R. (2013). Papeles de trabajo en la auditoria financiera (Tercera Edicion ed.). ECOE Ediciones.

Fierro A, Fierro F y Fierro F. (2016). Contabilidad de Pasivos, con estandares internacionales para pymes (Tercera ed.). Bogota: ECOE Edicinoes.

Fierro, A., \& Fierro, F. (2015). Contabilidad de activos con enfoque NIIF para las pymes (Tercera ed.). Bogota: ECOE Ediciones.

Fullana C y Paredes J. (2008). Manual de contabilidad de costes (Primera Edicion ed.). Madrid: Delta Publicaciones.

Guzmán , R., \& Verdezoto , M. (2015). Auditoría financiera y de cumplimiento legal Teoría y Práctica (Primera ed.). Machala: Utmach. 
Hoyos Ballesteros, R. (2009). La auditoria del servicio al cliente a través de la observación participante: "El cliente incógnito". Revista Colombiana de Marketing, 36.

Obtenido de http://www.redalyc.org/articulo.oa?id=10926778002

Lybrand, C. (1997). Nuevos conceptos del control interno. Madrid: Diaz de Santos.

Mantilla, S. (2008). AUDITORIA FINANCIERA PARA PYMES. Colombia: ECOEDICIONES.

Martinez, R. (2003). Auditoria de Estados Financieros,su proceso paso a paso (Primera ed.). El Salvador: Cultura S.A de C.V.

Mendebil, V. (2013). Elementos de Auditoria (Sexta Edicion ed.). Mexico: Cengage.

Morales, H. (2012). Introducción a la auditoria. Mexico: RED TERCER MILENIO S.C.

Osorio, I. (2000). Fundamentos de Auditoria de Estados Financieros. Mexico: Editorial Ecafsa.

Santillana, J. (2003). Auditoria Interna (Tercera ed.). Mexico: Pearson Educacion.

Obtenido de https://catedrafinancierags.files.wordpress.com/2012/04/auditoriainterna-juan-ramc3b3n-santillana.pdf

Sotomayor, A. (2008). Auditoria Administrativa, Proceso y aplicacion. Mexico: Litografica Ingramex.

Tapia C, G. E. (2016). Fundamentos de Auditoria, aplicacion practicas de las Normas Internacionales de Auditoria. Mexico: IMCP.

Tapia C, R. d. (2017). Autidoria interna, perspectivas de vanguardia (Primera Edicion ed.). Mexico: IMCP.

Vanegas, W. (2007). El trabajo de campo en auditoria (Septima ed.). Costa Rica: EditorialUniversidad estatal a distancia.

Vega, G., \& Madelayne, L. (2006). Las Auditorias de información en las organizaciones. Ciencias de la Información, 4. Obtenido de http://www.redalyc.org/articulo.oa?id=181418190001

Viloria, N. (2004). Una aproximación a un enfoque holístico en auditoria. Actualidad Contable Faces, 86. Obtenido de http://www.redalyc.org/articulo.oa?id=25700909 
Villardefrancos Álvarez, M. d., \& Rivera, Z. (2006). La auditoria como proceso de control: concepto y tipología. Ciencias de la Información, 53. Obtenido de http://www.redalyc.org/articulo.oa?id=181418190004

Zapata, G., \& Aymara, H. y. (2010). Reflecciones sobre la aplicación de las NIIF en la PYMES Venezolanas. Compendium, 25. Obtenido de http://www.redalyc.org/articulo.oa?id=88019355003 


\section{PARA CITAR EL ARTÍCULO INDEXADO.}

Pacheco Poma, W., Narváez Zurita, C., \& Erazo Álvarez, J. (2019). Procedimiento metodológico de auditoría interna para la Cooperativa de Ahorro y Crédito Santiago Ltda., del cantón Loja. Visionario Digital, 3(2.1.), 309-337.

https://doi.org/10.33262/visionariodigital.v3i2.1.557

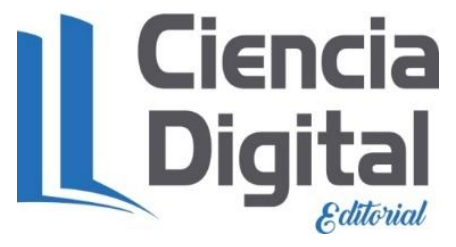

El artículo que se publica es de exclusiva responsabilidad de los autores y no necesariamente reflejan el pensamiento de la Revista Ciencia Digital.

El artículo queda en propiedad de la revista y, por tanto, su publicación parcial y/o total en otro medio tiene que ser autorizado por el director de la Revista Ciencia Digital.
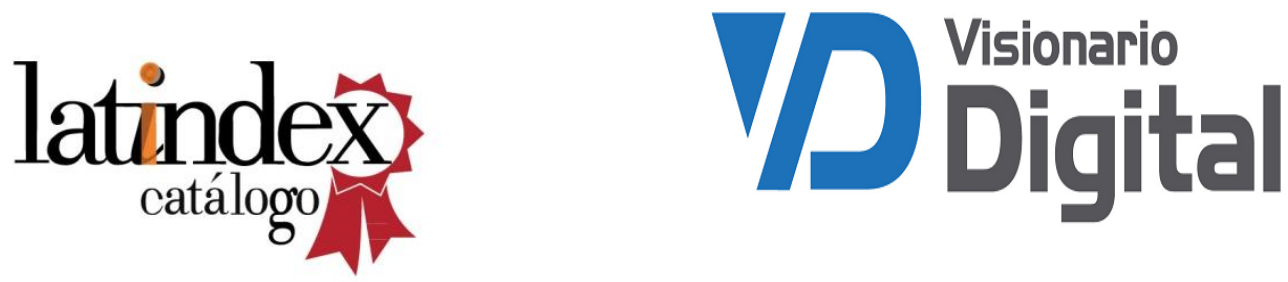